\title{
Formação de clusters na docência por interesse de pesquisa: método de auxílio à tomada de decisões em cursos de nível
} superior

\author{
Building teaching clusters by research interest: A method to assist the \\ decision making in higher education courses
}

\author{
Ricardo Brandão MANSILHA' ${ }^{1}$ (D) 0000-0002-7172-9881 \\ Miguel Afonso SELLITTO² (iD 0000-0002-8561-9085 \\ Daniel Pacheco LACERDA² (DD 0000-0002-8011-3376 \\ Rosiane SERRANO3 ${ }^{3}$ (D) 0000-0002-6618-0839
}

\begin{abstract}
Resumo
Este artigo apresenta o resultado da utilização de uma abordagem de análise multivariada e clusterização de docentes para tomada de decisões no curso de Engenharia de Produção em uma Instituição de Ensino Superior. Para isso, primeiramente, é realizada uma revisão conceitual sobre a importância da seleção e alocação de docentes nas diversas atividades de ensino, tais como bancas, orientações e atuação em sala de aula. Posteriormente, o presente estudo propõe um novo contexto de aplicação de ferramentas de clusterização, amplamente utilizadas em ambientes de manufatura, no contexto educacional, apresentando um método para clusterização dos docentes por temas de pesquisa, além do resultado prático da aplicação desse método em um curso de Engenharia de Produção. Por fim, são expostas algumas avaliações e considerações no intuito de contribuir com a gestão de cursos de nível superior, bem como de impulsionar e ampliar a base conceitual para aplicação dessa abordagem junto às instituições de ensino.
\end{abstract}

Palavras-chave: Análise Multivariada. Clusterização. Coeficiente de Similaridade. Seleção de Docentes.

\begin{abstract}
This article reports the results of a multivariate analysis application and teaching clusters on the decision-making in the Production Engineering course at a Higher Education Institution. For this purpose, firstly, a conceptual review was carried out on the importance of teacher selection and their allocation in different teaching activities, such as examining boards, orientations and lectures. Subsequently, the objective of the present study was to apply clustering tools, which are widely used in manufacturing environments, in an educational context, presenting a method of clustering for teachers by research topics, as well as the practical results of the

${ }^{1}$ Instituto Federal de Educação, Ciência e Tecnologia Farroupilha, Eixo Gestão de Negócios, Faculdade de Administração. Linha 7, s/n., Campus Frederico Westphalen, 98400-000, Frederico Westphalen, RS, Brasil. Correspondência para/Correspondence to: R.B. MANSILHA. E-mail:<ricardomansilha@gmail.com>.

${ }^{2}$ Universidade do Vale do Rio dos Sinos, Escola Politécnica, Programa de Pós-Graduação em Engenharia de Produção e Sistemas. São Leopoldo, RS, Brasil. ${ }^{3}$ Instituto Federal de Educação, Ciência e Tecnologia do Rio Grande do Sul, Eixo de Produção Cultural e Design, Faculdade de Tecnologia em Design de Moda. Erechim, RS, Brasil.

Recebido em 6 de setembro de 2017, versão final reapresentada em 28 de junho de 2018 e aprovado em 20 de julho de 2018.
\end{abstract}

Como citar este artigo/How to cite this article

Mansilha, R.B. et al. Formação de clusters na docência por interesse de pesquisa: método de auxílio à tomada de decisões em cursos de nível superior. Transinformação, v.30, n.3, p.287-298, 2018. http://dx.doi.org/10.1590/2318-08892018000300002 
application of this method in a course of Production Engineering. Finally, some evaluations and considerations are presented in order to contribute to the management of higher education courses, as well as to stimulate and broaden the conceptual basis for applying this approach in educational institutions.

Keywords: Multivariate Analysis. Clustering. Similarity Coefficient. Teacher Selection.

\section{Introdução}

O surgimento de um mundo conectado e interligado por redes de informação vem desafiando as organizações com dinâmicas mais complexas, cujas missões incluem relacionamentos imbricados com clientes e provedores de serviços. Tal situação vale para Instituições de Ensino Superior (IES). Caso uma IES possua recursos e capacidades sobrepujantes aos dos concorrentes, poderá criar vantagens competitivas (Nassif et al., 2010).

Estudos têm enfatizado a influência da diversidade do perfil e da qualidade técnica dos docentes como fatores cruciais no desempenho de uma IES no contexto brasileiro (Avelar et al., 2003; Pachane, 2003; Machado et al., 2011; Moita et al., 2015). Entretanto, não foram encontradas pesquisas que avaliem como combinar esses fatores nos processos de estruturação e seleção de docentes. Também não foram encontradas publicações a respeito de alocações docentes para bancas, orientações ou atuação em salas de aula com base em ferramentas e técnicas estruturadas de apoio à decisão. Assim, justifica-se o esforço no sentido de direcionar pesquisas para ampliar o conjunto de ferramentas e técnicas que sirvam de auxílio à tomada de decisão nas IES.

Dessa forma, o objetivo deste artigo consiste em proceder a uma aplicação prática do processo de clusterização para tomada de decisões no que diz respeito à alocação do quadro docente em cursos de graduação, considerando as diferentes especificidades e atividades docentes desenvolvidas nas IES. Busca-se, assim, um método que possa auxiliar as IES quanto à organização e melhoria da gestão docente em cursos de graduação. Para o alcance do objetivo proposto, tomou-se como base o estudo de caso de um curso de graduação de Engenharia de Produção, ofertado por uma universidade privada localizada no sul do Brasil.

\section{Seleção de Docentes}

O Brasil tem vivenciado um período de alterações no sistema de ensino superior, que englobam crescimento, expansão, flexibilização, novos critérios de ingresso, exigências de titulações do corpo docente e mecanismos de avaliação diferenciados (Cobbe et al., 2015). No contexto brasileiro, as IES consistem, predominantemente, em empresas privadas, nas quais a qualidade do corpo discente, a taxa de matrícula e a qualidade docente são relevantes para determinar a demanda de mercado (Moita et al., 2015).

Nesse cenário, ao selecionar um docente, são priorizadas competências relacionadas à transmissão de conhecimento e à realização de atividades práticas (Machado et al., 2011). Para uma seleção docente eficaz, avaliam-se a consonância entre o cargo e os valores individuais dos candidatos, ou seja, se estes se aproximam dos valores institucionais (Avelar et al., 2003).

No Brasil, os docentes não possuem uma identidade única, assim como não há um sistema de educação superior unificado. Existem docentes oriundos de cursos de licenciatura, outros advindos do mercado de trabalho e da formação em pós-graduações, com ou sem experiência prévia no ambiente de ensino ou no âmbito profissional de forma geral (Machado et al., 2011). Contudo, observa-se que as características docentes necessitam extrapolar os limites do conhecimento proveniente de especializações e de habilidades de pesquisa, o que é evidenciado, por exemplo, nas orientações realizadas pelos docentes, uma vez que as trajetórias dos orientandos não necessariamente se coadunam com sua especialidade ou linha de pesquisa (Pachane, 2003). 
Desse modo, a capacidade de adaptação do docente universitário pode definir sua identidade, determinando um desempenho de excelência (Machado et al., 2011). Portanto, visando minimizar a incidência de casos de alocação de docentes em áreas não condizentes com seus interesses de pesquisa, buscam-se, cada vez mais, métodos de seleção e encaminhamento dos docentes a atividades de ensino, tais como o desenvolvido por esta pesquisa.

\section{Análise de Agrupamentos}

A análise de agrupamentos é uma forma de reunir um conjunto de objetos de tal maneira que os semelhantes entre si sejam reunidos por clusters (Benabdeslem; Bennani, 2006). Por conseguinte, objetos similares entre si devem estar juntos, bem como separados de outros objetos que também sejam similares entre si, porém diferentes dos primeiros (Sharma; Yadav, 2013).

Assim, a formação de clusters ou de aglomerados é uma técnica de análise multivariada que permite reunir objetos segundo características comuns (Gusberti et al., 2014). Amplamente utilizados no ambiente de manufatura (Seifoddini, 1989), os clusters são grupos criados para unir objetos ou indivíduos, conforme suas similaridades, por meio de critérios previamente estabelecidos (Corrar et al., 2007). Elementos que mais se assemelhem devem permanecer em um mesmo grupo (Meyer, 2002), devendo, portanto, ser abordados por uma estratégia comum (Niwattanakul et al., 2013).

Para a formação de clusters, são necessários, dessa forma, critérios de alocação. A literatura propõe coeficientes de similaridade (Yin, 2006), desenvolvidos com o objetivo específico de se adaptar a situações particulares e de interesse dos pesquisadores (Meyer, 2002). Esses coeficientes indicam o grau de semelhança entre pares de objetos, facilitando o processo de clusterização dos mais variados tipos de elementos (Yin, 2006).

Tais coeficientes se dividem em duas categorias: medidas de similaridade e de dissimilaridade. Na primeira, quanto maior for a semelhança, maior será o coeficiente; na segunda, quanto menor for a semelhança, menor será o coeficiente (Meyer, 2002; Yin, 2006; Reina et al., 2014). Além disso, coeficientes de similaridade podem variar conforme as características de interesse do objeto observado, expressas em variáveis que podem ser contínuas, discretas ou binárias (Reina et al., 2014).

Posto que a busca por similaridades ou dissimilaridades entre elementos de um mesmo grupo pode ser realizada de diferentes maneiras, a primeira providência para a formação de um cluster é a definição dos critérios que serão utilizados para a segregação desses grupos (Meyer, 2002). Nesse sentido, diferentes coeficientes encontram-se disponíveis na literatura (Meyer, 2002; Pachane, 2003; Yin, 2006). Dentre eles, segundo Yin (2006), podem ser citados os coeficientes de Pearson; Jaccard; Czekanowsky; Kulczynski; Russel e Rao; Sorensen-Dice; Ochiai; Yule; Rogers e Tanimoto; Hamann; Sokal e Sneath; e Anderberg.

Depois de calculados, os coeficientes devem ser organizados de modo a facilitar a clusterização. Para auxiliar nesse processo, pode ser utilizada a plotagem dos valores oriundos dos resultados de similaridade no formato de dendogramas (Krivánek, 1986). O dendograma é uma ferramenta usual de representação dos resultados de algoritmos hierárquicos, que demonstra intuitivamente a ordem do agrupamento (Linden, 2009). Embora os dendogramas possam apresentar algumas limitações, pois não são abordagens exatas (Schonlau, 2002), por seu intermédio, se o número de variáveis não for extenso, é possível alcançar resultados satisfatórios (Metz, 2006).

\section{Procedimentos Metodológicos}

Em pesquisas na área da Ciência da Informação, o rigor de um trabalho científico depende do tipo de estratégia metodológica utilizada. A definição dessas estratégias, que dizem respeito a domínios políticos e epistemológicos ancorados em suas estruturas, além de amparar, proporciona as condições necessárias para produção em matéria de pesquisa (González de Gómez, 2000). 
Nesse sentido, a estratégia metodológica adotada para o desenvolvimento desta pesquisa foi um estudo de caso único. Os estudos de casos são adequados para a compreensão em profundidade de assuntos aplicados e pouco explorados (Dubé; Paré, 2003). Além do mais, o estudo de caso pode ser conduzido pela abordagem tanto qualitativa quanto quantitativa (Eisenhardt, 1989). Piran et al. (2017), por exemplo, utilizaram uma combinação de ferramentas estatísticas para analisar os efeitos da modularização sobre a eficiência dos processos produtivos em uma fabricante de ônibus brasileira. A abordagem quantitativa é mais apropriada para casos únicos, pois possui maior potencial quanto à validade interna dos resultados (Voss et al., 2002; Barrat et al., 2011), tendo sido, portanto, empregada nesta pesquisa.

Estudos exploratórios, como este, concentram-se normalmente na resolução de problemas específicos do cotidiano organizacional e/ou institucional (Freitas et al., 2016). Assim, a partir de um estudo de caso único, buscou-se explorar o processo de análise de clusters como auxílio para tomada de decisões em problemas práticos designados a coordenações de cursos de graduação. O estudo de caso ocorreu no curso de bacharelado de Engenharia de Produção de uma instituição privada localizada no estado do Rio Grande do Sul, abarcando análises documentais, averiguação de cadastros na plataforma Lattes e entrevistas informais junto aos coordenadores do curso em questão.

Para efetuar a investigação proposta, a primeira etapa do método de trabalho foi identificar os docentes atuantes exclusivamente na Engenharia de Produção que atendessem diretamente à coordenação do curso. Após segregar os docentes participantes, o currículo Lattes de cada um foi analisado com a finalidade de classificá-lo conforme suas publicações. Tais informações foram tabuladas em uma matriz com auxílio de planilhas do Excel.

Após, foram selecionados coeficientes para o cálculo de similaridade. Esses coeficientes auxiliaram na percepção quanto ao grau de semelhança entre os docentes. Assim, a partir dos resultados obtidos pelo cálculo dos coeficientes e com o auxílio do software Minitab, foram gerados gráficos conhecidos por dendogramas. Com os dendogramas plotados, foi possível formar os clusters dos docentes por similaridade relativa aos temas de pesquisa, gerando, assim, uma nova matriz para organizar os docentes em seus respectivos clusters.

Contudo, durante o processo de avaliação dos resultados da presente pesquisa, surgiu a necessidade de adaptar os resultados finais às particularidades do curso analisado. Nesse processo, foi sugerida a construção de um quadro-síntese que definisse quais docentes eram responsáveis por quais temas em específico. Tal avaliação foi realizada pela coordenação do referido curso a partir de dois encontros: o primeiro teve a finalidade de estudar os resultados, formar opiniões e discutir melhorias; e o segundo, de discutir as considerações finais a respeito do que foi produzido.

Na seção seguinte, a aplicação prática do processo de clusterização, assim como o método de elaboração deste trabalho, é detalhada passo a passo. Dessa forma, os critérios de escolha, os dados coletados e a lógica de construção desta pesquisa podem ser visualizados a seguir.

\section{Matriz de Posicionamento e Coeficientes de Similaridade}

Os dados coletados na plataforma Lattes e tabulados na matriz de posicionamento são apresentados no Quadro 1. A matriz de posicionamento relaciona os docentes com os temas de pesquisa identificados na plataforma. Nas colunas, são expostos 26 temas encontrados nos currículos dos docentes e, nas linhas, são apontados os docentes exclusivos do bacharelado de Engenharia de Produção. Nesse processo, foram identificados 23 docentes (de um total de 86 membros do colegiado) que atuam nas áreas exclusivas do curso em questão.

Após, foram selecionados três coeficientes de similaridade e calculados os fatores a partir desses coeficientes: (i) Jaccard; (ii) Yule; e (iii) Ochiai. Enquanto os coeficientes Yule e Ochiai foram eleitos aleatoriamente, o coeficiente Jaccard foi designado por possuir maior destaque na literatura. A seguir, constam as fórmulas de cada coeficiente. 
Quadro 1. Matriz de Posicionamento dos Temas de Pesquisa por Docente.

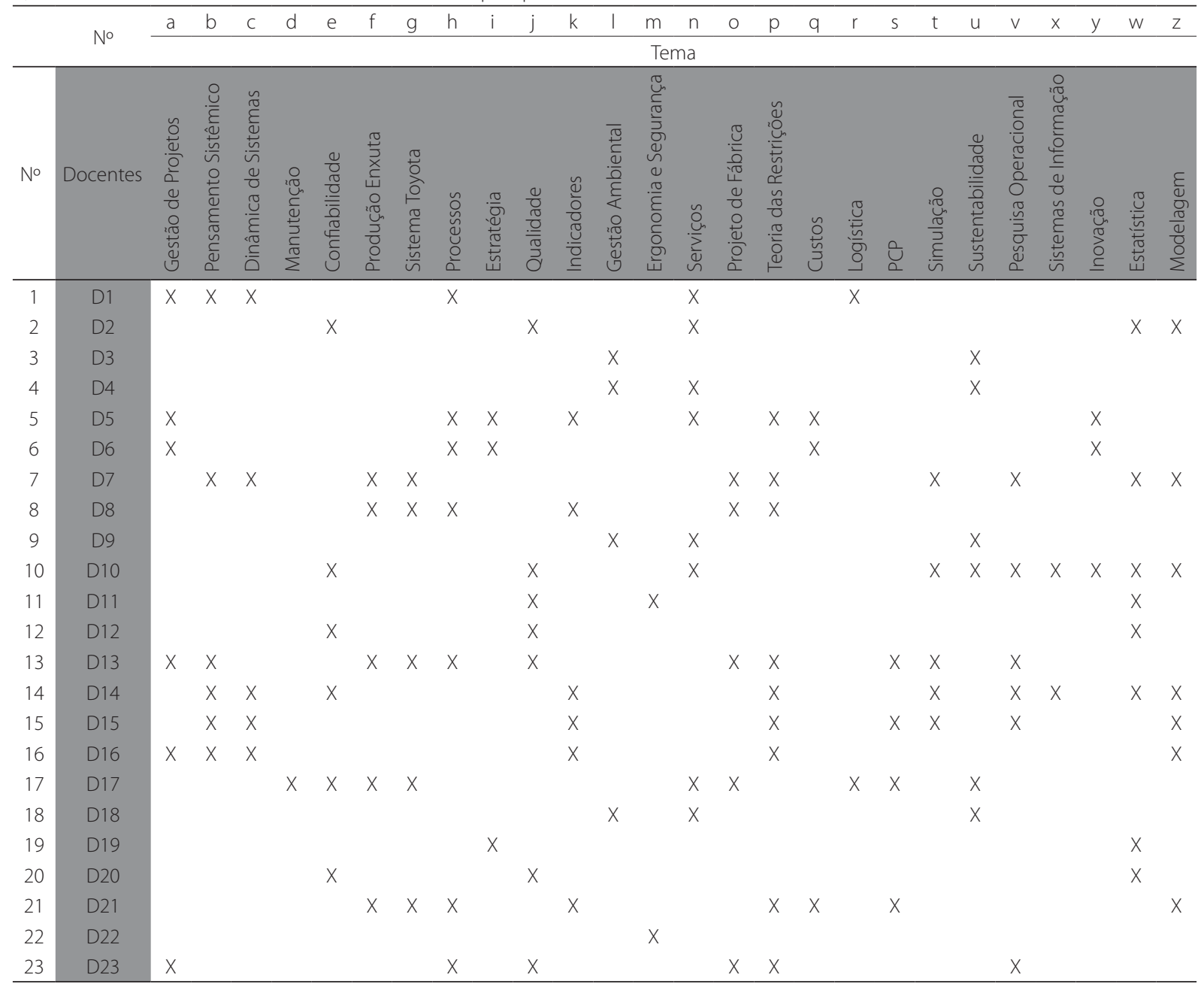

Fonte: Elaborado pelos autores (2016).

$$
\begin{aligned}
& \mathrm{Sij}=\frac{\mathrm{a}}{\mathrm{a}+\mathrm{b}+\mathrm{c}} \\
& \mathrm{Sij}=\frac{\mathrm{ad}-\mathrm{bc}}{\mathrm{ad}+\mathrm{bc}} \\
& \mathrm{Sij}=\sqrt{\frac{\mathrm{a}}{[(\mathrm{a}+\mathrm{b})(\mathrm{a}+\mathrm{c})]}}
\end{aligned}
$$

Sendo:

$\mathrm{a}=$ número de temas abordados por ambos os docentes, i e j;

$\mathrm{b}=$ número de temas abordados apenas pelo docente i;

$c=$ número de temas abordados apenas pelo docente j;

$\mathrm{d}=$ número de temas não abordados por ambos os docentes, i e j.

Tendo em vista a quantidade de cálculos efetuados para obter os coeficientes pareados entre os docentes, não serão apresentadas as planilhas de informações reveladas pelos três coeficientes. Apesar disso, os resultados calculados podem ser visualizados por intermédio dos dendogramas exibidos, na Figura 1. Os resultados dos dendogramas mostram maior semelhança entre os coeficientes de Jaccard (i) e Yule (ii) e menor semelhança entre estes e o coeficiente de Ochiai (iii). 

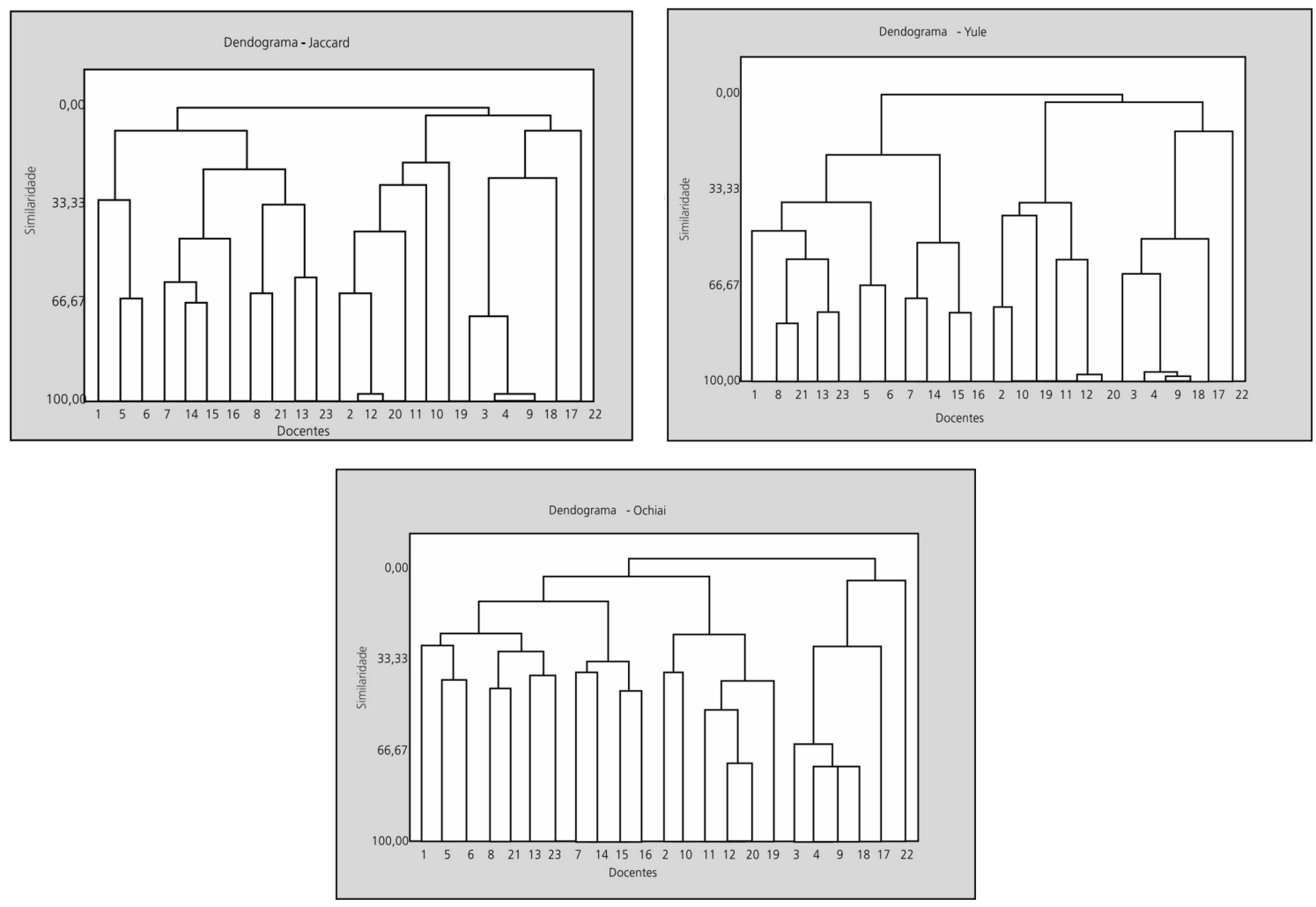

Figura 1. Resultado dos Dendogramas.

Fonte: Elaborado pelos autores (2016).

Na tentativa de organizar as informações dos dendogramas, os resultados foram arranjados e classificados na Tabela 1. Observa-se que os resultados dos Grupos 1 a 6 foram idênticos para os três coeficientes. Nos Grupos 7 e 8, somente os coeficientes de Jaccard (i) e Yule (ii) apresentaram interesses iguais. O Grupo 9, por sua vez, mostrou semelhança entre Yule (ii) e Ochiai (iii), enquanto o Grupo 10 apontou resultados distintos nos três coeficientes quanto aos temas de pesquisa.

Tabela 1. Síntese dos Resultados.

\begin{tabular}{lcccccccccc}
\hline \multicolumn{1}{c}{ Coef. } & G1 & G2 & G3 & G4 & G5 & G6 & G7 & G8 & G9 & G10 \\
\hline Jaccard & 1 & $5 ; 6$ & $7 ; 14 ; 15 ; 16$ & $3 ; 4 ; 9 ; 18$ & 17 & 22 & $8 ; 21 ; 13 ; 23$ & 19 & 10 & $2 ; 12 ; 20 ; 11$ \\
Yule & 1 & $5 ; 6$ & $7 ; 14 ; 15 ; 16$ & $3 ; 4 ; 9 ; 18$ & 17 & 22 & $8 ; 21 ; 13 ; 23$ & 19 & $2 ; 10$ & $11 ; 12 ; 20$ \\
Ochiai & 1 & $5 ; 6$ & $7 ; 14 ; 15 ; 16$ & $3 ; 4 ; 9 ; 18$ & 17 & 22 & $8 ; 21$ & $13 ; 23$ & $2 ; 10$ & $11 ; 12 ; 20 ; 19$ \\
\hline
\end{tabular}

Fonte: Elaborado pelos autores (2016).

Depois de comparar o quadro-síntese (Tabela 1) com a matriz de posicionamento (Quadro 1), alguns ajustes foram necessários para adaptar os resultados obtidos à realidade do curso referido. O principal ajuste realizado foi a redução de dez para sete grupos de docentes, já que, no processo de clusterização, os dendogramas apresentaram três grupos formados por um único docente. Consequentemente, os grupos formados por apenas um docente foram eliminados, e os docentes, realocados nos grupos permanecentes.

Definidos os grupos, a matriz de resultados foi concebida para divulgação final dos clusters encontrados (Quadro 2). Essa matriz organiza as informações referentes aos grupos sugeridos, considerando o número de docentes alocados de acordo com o tema e o total de docentes disponíveis que trabalham nas respectivas áreas. 
Quadro 2. Matriz de Resultados.

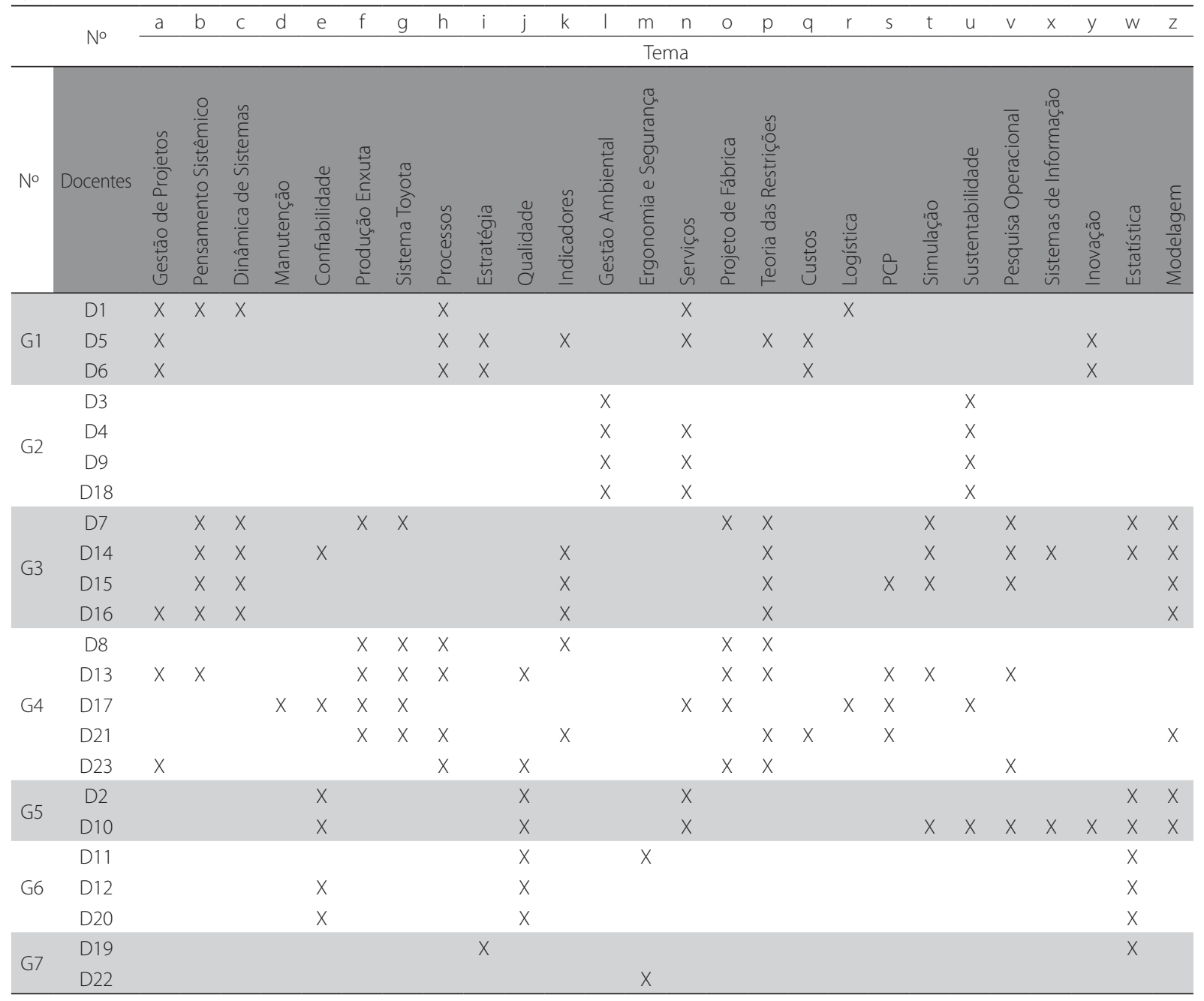

Fonte: Elaborado pelos autores (2016).

Conforme pode ser observado no Quadro 2, os resultados apresentados sugerem grupos de docentes com certa semelhança relativa aos interesses de pesquisa. Entretanto, não esclarecem quais são os grupos e docentes responsáveis por cada um dos temas expostos pela matriz. Nota-se, assim, que não foi possível definir grupos exclusivos para cada um dos temas, já que os interesses são diversos e variam de docente para docente - dificilmente, os interesses de mais de um docente pela pesquisa são exatamente iguais. No Grupo 2, por exemplo, os docentes D4, D9 e D18 possuem exatamente a mesma marcação na matriz de resultados (Quadro 2); entretanto, o docente D3, que também pertence ao Grupo 2, difere dos demais por não compactuar com o tema "serviços".

Dessa maneira, além de sintetizar os grupos e docentes por áreas de interesse, o Quadro 2 organiza e expõe informações para auxiliar a coordenação no processo de tomada de decisões quanto à alocação dos docentes nas mais diversas atividades de ensino. Essas informações foram organizadas junto aos coordenadores do curso de Engenharia de Produção, que sugeriram como resultado final a distribuição em número de docentes por tema de pesquisa.

Observa-se, a partir do Quadro 2, que, para cada assunto relacionado ao curso, é possível alocar os docentes individualmente ou em grupo. Enquanto a coluna denominada "Alocados" mostra a quantidade de docentes responsáveis por atender aos temas em um contexto específico, a coluna "Sem Publicação" expõe a quantidade de docentes alocados que não apresentaram índices de pesquisa em seus currículos. Já a coluna "Excedentes" diz respeito 
ao número de docentes que não foram alocados em determinado tema, mas possuem interesse por determinado assunto, conforme seus currículos. Por último, no item "Total", é visualizado o somatório entre os docentes alocados e os excedentes.

Dessarte, a Tabela 2 sintetiza os dados da matriz de resultados (Quadro 2) em informações para a coordenação do curso de Engenharia de Produção. O tema "estratégia", por exemplo, é composto pelo docente D19 em conjunto com o Grupo 1, que possui três integrantes. Entretanto, o docente D1, pertencente ao Grupo 1, não possui publicações na área de estratégia.

Logo, ao verificar a necessidade de alocar um docente fora da área de domínio, sugere-se que a busca seja feita junto aos grupos predefinidos. Conforme exemplo exposto pelo tema "estratégia", mesmo que o docente D1 não possua publicações na área, caso necessário, este seria contatado, pois seu perfil é o mais próximo dos docentes vinculados ao Grupo 1.

Tabela 2. Temas por Grupos.

\begin{tabular}{|c|c|c|c|c|c|}
\hline Interesse de Pesquisa & Grupos e Docentes & Alocados & Sem Publicação & Excedentes & Total \\
\hline Manutenção & D17 e D22 & 2 & 1 & 0 & 2 \\
\hline Ergonomia e Segurança & D22 e D11 & 2 & 0 & 0 & 2 \\
\hline Logística & D1 e D17 & 2 & 0 & 0 & 2 \\
\hline Sistemas de Informação & D14 e D10 & 2 & 0 & 0 & 2 \\
\hline Estratégia & $\mathrm{G} 1+\mathrm{D} 19$ & 4 & 1 & 0 & 4 \\
\hline Custos & $\mathrm{G} 1+\mathrm{D} 21$ & 4 & 1 & 0 & 4 \\
\hline Inovação & $\mathrm{G} 1+\mathrm{D} 10$ & 4 & 1 & 0 & 4 \\
\hline Gestão Ambiental & G2 & 4 & 0 & 0 & 4 \\
\hline PCP & $\mathrm{G} 4+\mathrm{D} 15$ & 6 & 2 & 0 & 6 \\
\hline Dinâmica de Sistemas & G3 & 4 & 0 & 1 & 5 \\
\hline Produção Enxuta & G4 & 5 & 1 & 1 & 6 \\
\hline Sistema Toyota & G4 & 5 & 1 & 1 & 6 \\
\hline Projeto de Fábrica & G4 & 5 & 1 & 1 & 6 \\
\hline Simulação & $\mathrm{G} 3+\mathrm{D} 10$ & 5 & 1 & 1 & 6 \\
\hline Gestão de Projetos & $\mathrm{G} 1+\mathrm{D} 13$ & 4 & 0 & 2 & 6 \\
\hline Pensamento Sistêmico & G3 & 4 & 0 & 2 & 6 \\
\hline Confiabilidade & G5 e G6 & 5 & 1 & 2 & 7 \\
\hline Indicadores & $\mathrm{G} 3+\mathrm{D} 5$ & 5 & 1 & 2 & 7 \\
\hline Sustentabilidade & G2 & 4 & 0 & 2 & 6 \\
\hline Pesquisa Operacional & $\mathrm{G} 3+\mathrm{D} 23$ & 5 & 1 & 2 & 7 \\
\hline Processos & G4 & 5 & 1 & 3 & 8 \\
\hline Qualidade & G5 e G6 & 5 & 0 & 2 & 7 \\
\hline Modelagem & G3 e G5 & 6 & 0 & 1 & 7 \\
\hline Serviços & G2 e G5 & 6 & 1 & 3 & 8 \\
\hline Estatística & G6 & 4 & 0 & 4 & 8 \\
\hline Teoria das Restrições & $\mathrm{G} 4+\mathrm{D} 5$ & 6 & 1 & 4 & 10 \\
\hline
\end{tabular}

Fonte: Elaborado pelos autores (2016).

\section{Discussão}

Este estudo apresenta uma aplicação prática para a formação de clusters de docentes conforme interesses de pesquisa. Os resultados dessas aplicações podem servir de auxílio à tomada de decisões, especialmente para gestores de cursos de graduação. Embora a ferramenta de clusterização possa ser utilizada em outros níveis na área de ensino, aparentemente, o impacto na gestão de cursos de graduação é maior. Isso ocorre porque, nos cursos de pós-graduação, os programas disponibilizam, com antecedência, um número limitado de vagas direcionadas a temas 
específicos de pesquisa. Além disso, nos níveis de ensino médio e fundamental, também é necessário apresentar títulos nas áreas específicas para ministrar as aulas e não existe a necessidade de participar de bancas e orientações.

Dessa maneira, como suporte para tomada de decisões, principalmente voltada à gestão e coordenação dos cursos de graduação, o presente estudo mostrou-se oportuno por inúmeras razões. A primeira refere-se ao auxílio no planejamento de oferta de disciplinas, que geralmente ocorre nas instituições de ensino periodicamente. Nesse planejamento, a coordenação de curso é responsável por ofertar as disciplinas aos discentes, disponibilizando docentes com conhecimento na área para ministrar as aulas, sejam estes do quadro atual ou oriundos de novas alocações.

Nos cursos de graduação, o planejamento da oferta de disciplinas é dinâmico, de modo que a alocação do quadro docente depende do número de discentes ingressantes, dos possíveis egressos do curso a cada período, da quantidade de discentes matriculados ao longo do curso e da própria disponibilidade dos docentes. Ademais, algumas disciplinas podem ser anualizadas e outras subdivididas em mais de uma turma. Por essa razão, a distribuição das disciplinas e do quadro de horários, bem como a alocação docente, costuma ser diferente de uma oferta para outra, de forma que os docentes são constantemente realocados para diferentes atividades.

Variáveis externas também podem influenciar esse arranjo, tais como contratação e/ou desligamento de docentes da instituição de ensino. Além disso, existem casos de afastamento parcial dos docentes, tais como as licenças concedidas por enfermidade, gestação e qualificação. Essas variáveis repercutem na necessidade de uma revisão periódica do plano de oferta das disciplinas, a partir de uma reavaliação do quadro docente, considerando suas disponibilidades e experiências.

Ressalta-se, ainda, que, em cursos de graduação, outros dois pontos são considerados na alocação dos docentes: a formação das bancas dirigidas aos Trabalhos de Conclusão de Curso (TCC) e a busca por docentes que possam orientar esses trabalhos. Enquanto a escolha por orientadores ocorre aleatoriamente de acordo com o interesse de pesquisa dos discentes, a composição das bancas de graduação costuma alocar pelo menos três docentes com conhecimento na área. Dessa maneira, a formação das bancas depende do conhecimento dos docentes quanto aos temas e assuntos que serão tratados pelos discentes em conjunto com seus orientadores.

Assim, a matriz de resultados (Quadro 2) também visa facilitar o processo de busca do orientador por parte dos discentes. Embora, em muitos casos, a escolha de um orientador ocorra em sala de aula, por afinidade com o tema discutido ou mesmo por empatia com determinado docente, a busca junto à coordenação também acontece, principalmente quando o curso é composto por muitos discentes e uma gama elevada de docentes.

Nesse caso, o estudo poderia se resumir à matriz de posicionamento (Quadro 1), por administração visual. Como solução, o discente percorreria na matriz a coluna referente ao tema de interesse e, então, verificaria os docentes vinculados a cada um dos temas. Contudo, nem sempre os docentes se encontram disponíveis. Outras vezes, mesmo que haja disponibilidade, o discente, por motivos particulares, pode demonstrar desinteresse em ser orientado por determinado docente. Nesse sentido, muitas vezes, é preciso indicar um orientador que não esteja vinculado ao tema sugerido pelo discente.

Já em relação à formação das bancas, devido à quantidade de docentes disponíveis por área e à disponibilidade de agenda destes, nem sempre é possível formar bancas com docentes que possuam experiência sobre o assunto que será tratado. Nessa situação, o ideal seria dispor de um número considerável de docentes para cada um dos assuntos tratados durante o curso. Todavia, como é necessário racionar o número de docentes contratados em virtude do custo implicado pela folha de pagamento, a solução proposta pela clusterização seria promover a capacitação interna dos docentes em longo prazo, por intermédio do compartilhamento de experiência e saber entre os integrantes de cada grupo formado.

A ideia é que os clusters possam aproximar os docentes e que estes consigam evoluir no que concerne ao conhecimento acerca de determinados assuntos à medida que vão estudando os temas tratados pelos grupos 
em que atuam, uma vez que é natural que os docentes sejam alocados em disciplinas nunca lecionadas antes ou que participem de bancas cujo assunto não é de seu domínio. Sendo assim, o ideal seria alocar sempre os mesmos docentes para determinados assuntos. Isso levaria, ao longo do tempo, à capacitação dos docentes sobre os temas pertinentes ao grupo de que fazem parte.

Nesse sentido, ao passo que novas ofertas, bancas e orientações fossem ocorrendo, os docentes seriam instigados a ler e estudar sobre os temas tratados pelos grupos. Deve-se considerar, ainda, que, geralmente, os docentes atuantes exclusivamente nos cursos de graduação são contratados por possuir formação condizente com as respectivas competências. Além disso, embora alguns docentes não tenham interesse por determinados assuntos, isso não significa que eles desconheçam por completo tais temas. Nesse contexto, a clusterização dos docentes por interesses de pesquisa poderia refinar as competências do curso com o passar do tempo.

Outra situação em que é necessário tomar decisões referentes ao perfil dos docentes na graduação ocorre no momento da contratação, ainda que, normalmente, os docentes sejam contratados para lecionar em disciplinas específicas. Nesses casos, entrevistas e análises curriculares são concebidas para verificar a didática e a adequação do docente às disciplinas para as quais está sendo contratado. Contudo, uma análise aprofundada quanto ao interesse de pesquisa poderia ser interessante, visto que, futuramente, possibilitaria verificar e sustentar os grupos e até mesmo contratar novos docentes com habilidades certeiras para cada cluster.

Faz-se necessário, entretanto, frisar algumas limitações desse processo de clusterização dos docentes por tema como ferramenta para tomada de decisões. O primeiro problema encontra-se no fato de que, muitas vezes, a rotatividade dos docentes em algumas instituições é alta. Embora as matrizes e os cálculos possam ser automatizados em planilhas, a coleta dos dados de entrada, a plotagem dos dendogramas e a posterior análise dos dados de saída precisam ser efetuadas manualmente e podem exigir muito tempo.

O segundo inconveniente é que, muitas vezes, na contratação dos docentes, o perfil dos candidatos não supre a expectativa do processo seletivo. Dependendo da região onde as instituições estão inseridas, existe certa dificuldade na seleção dos docentes devido ao baixo número de candidatos. Nessas situações, outros critérios precisam ser levados em consideração.

Ademais, nas instituições governamentais, a seleção é feita por intermédio de concursos públicos, o que implica aceitar o docente que alcançou o primeiro lugar independentemente de seu perfil condizer ou não com os grupos formados pela ferramenta de clusterização. Para isso, seria necessária uma descrição mais elaborada como critério para inscrição dos candidatos aos processos seletivos.

Por último, é preciso considerar que a tabulação inicial exclusivamente por meio da plataforma Lattes pode não representar o verdadeiro perfil dos docentes, já que alguns profissionais costumam não publicar artigos frequentemente e, quando o fazem, nem sempre são os autores principais dessas publicações. Assim, é possível afirmar que a tarefa mais complexa dessa aplicação consiste na configuração inicial da matriz de posicionamento. Se, por um lado, as publicações que constam no currículo dos docentes podem não representar o verdadeiro interesse de pesquisa, por outro lado, ao entrevistar esses colaboradores como solução, corre-se o risco de que estes deixem de mencionar alguma área de domínio por motivos pessoais, visto que algumas disciplinas exigem mais esforço para serem conduzidas do que outras.

\section{Conclusão}

O gerenciamento nas instituições de ensino torna-se, com o passar do tempo, mais dinâmico e complexo, pois elas se encontram inseridas em um ambiente cada vez mais competitivo, em que o ensino é construído tanto de forma presencial quanto a distância. Desse modo, é preciso reavaliar as competências, reduzir os custos e proporcionar a entrega dos serviços com qualidade superior à da concorrência.

A principal fonte de renda de faculdades, centros universitários e universidades, geralmente, advém dos cursos de graduação. Logo, a gestão dos cursos de bacharelado e licenciatura precisa adaptar-se à realidade competitiva 
em que se encontram. Para isso, é necessária uma excelente equipe de profissionais que possam suprir as exigências dos discentes. Contudo, quando o foco se encontra na gestão dos cursos, tal exigência vai além da didática e do conhecimento sobre determinado assunto em sala de aula: espera-se do corpo docente flexibilidade quanto às modalidades de ensino e ao número de disciplinas de domínio.

Posto que o quadro docente precisa ser enxuto para que as instituições consigam competir no que concerne aos custos repassados aos discentes, o ideal é que os docentes sejam flexíveis. Essa flexibilidade inclui a capacidade de transitar pelas disciplinas exclusivas do curso. O docente precisa, assim, atender ao maior número de disciplinas possíveis, devendo, para isso, dominar diversos assuntos. Quanto mais assuntos forem de domínio de determinado docente, maior será sua flexibilidade para fazer frente às diferentes atividades exigidas para a composição do currículo do curso.

Além do exercício em sala de aula, na graduação exigem-se dos docentes orientações dos trabalhos de conclusão, participações em bancas, realização de projetos de extensão etc. No entanto, essas atividades são estendidas a cada uma das disciplinas específicas do curso, tornando complexa a gestão do quadro docente.

Um docente que ministra a disciplina de Administração da Produção, por exemplo, deve possuir, preferencialmente, conhecimento prático e teórico sobre o assunto e disponibilidade para orientar e avaliar TCC sobre diversos temas atinentes a essa disciplina. Assim, um docente que assume mais de uma disciplina - e isso é comum nas instituições - precisa ser flexível o suficiente para dominar mais de uma área do conhecimento, pois cada disciplina demanda conhecimento amplo sobre diversos assuntos que deverão ser tratados em sala de aula e em orientações dos discentes.

Dessa maneira, a formação de clusters de docentes por assuntos de interesse mostrou-se satisfatória. Como decorrência deste estudo, os resultados da aplicação prática realizada foram compartilhados com a coordenação do curso de Engenharia de Produção, chamando a atenção principalmente quanto à carência de profissionais para tratar de alguns assuntos específicos. A formação de clusters, além de beneficiar os discentes quanto à identificação de seus futuros orientadores e auxiliar na gestão do curso, gera um respaldo holístico em relação ao processo de contratação de novos profissionais.

O quadro-síntese de temas por grupos (Quadro 2) passou a ser utilizado pela instituição nas reuniões iniciais para discentes matriculados em TCC. Sendo assim, a gestão visual facilitou a busca por orientadores, reduzindo o número de discentes que procuravam a coordenação para auxiliar nesse processo. Entretanto, a fim de facilitar ainda mais o processo de busca, seriam necessárias informações atualizadas no que diz respeito ao número de orientandos alocados a cada um dos orientadores, pois é natural que exista uma limitação quanto ao número de orientandos que cada docente pode orientar ao mesmo tempo. Caso não seja respeitada tal restrição, entende-se que as orientações poderão perder qualidade.

Ademais, a formação de bancas pela coordenação do curso, que, no caso avaliado, também é responsável por gerenciar as atividades de TCC, tornou-se mais simples. Isso de deve ao fato de que a formação dos grupos, além de aumentar a disponibilidade de docentes por assunto, tornou transparente a visualização dos assuntos de interesse de cada um.

Diante disso, como proposta futura, sugere-se a clusterização por meio de matrizes de posicionamento, formadas a partir de entrevistas com os docentes e de pesquisas diretamente na plataforma Lattes. Nesse caso, seria necessário avaliar se o posicionamento em relação às áreas se tornaria mais preciso para a formação dos clusters e se a presença de entrevistas não aumentaria a distorção dos dados, tendo em vista que determinados assuntos podem ser evitados pelos docentes por motivos pessoais.

Outra sugestão seria testar diferentes coeficientes para verificar se estes poderiam ser utilizados no contexto das instituições de ensino - um estudo em que o foco não estaria na aplicação, mas em entender quais seriam os coeficientes mais indicados para esse contexto. Há, também, a possibilidade de testar a ferramenta de clusterização como proposta para capacitação dos grupos, o que não chegou a ser feito neste estudo. Para isso, seria necessário aplicar os passos desta pesquisa e avaliar, em longo prazo, o comportamento e a evolução do quadro docente para identificação dos resultados. 


\section{Colaboradores}

Com exceção da tabulação dos dados, que foi feita pelo primeiro autor, todos os autores contribuíram em todas as etapas do artigo.

\section{Referências}

Avelar, L.P.et al. Seleção de professores: uma experiência inovadora. Psicologia Escolar educacional, v.7, n.2, p.217-219, 2003.

Barratt, M.; Choi, T.; Li, M. Qualitative case studies in operations management:Trends, research outcomes, and future research implications. Journal of Operations Management, v.29, n.4, p.329-342, 2011

Benabdeslem, K.; Bennani, Y. Dendogram-based SVM for multi-class classification. Journal of Computing and Information Technology, v.14, n.4, p.283-289, 2006.

Cobbe, P.R.C.O. et al. A inteligência organizacional como instrumento de autoavaliação em instituições de ensino superior. Perspectivas em Gestão \& Conhecimento, v.5, n.2, p.111-126, 2015.

Corrar, L.J. et al. Análise multivariada para os cursos de Administração, Ciências Contábeis e Economia. São Paulo: Atlas, 2007.

Dubé, L.; Paré, G. Rigor in information systems positivist case research: Current practices, trends, and recommendations. Mis Quarterly, v.27, n.4, p.597-636, 2003.

Eisenhardt, K.M. Building theories from case study research. The Academy of Management Review, v.14, n.4, p.532-550, 1989.

Freitas. L.J.; Bufrem, L.S.; Breda, S.M. Methodological choices for research in Information Science: Contributions to domain analysis. Transinformação, v.28, n.1, p.5-13, 2016. http://dx.doi. org/10.1590/2318-08892016002800001

González de Gómez, M.N. Metodologia de pesquisa no campo da Ciência da Informação. DataGramaZero, v.1, n.6, 2000.

Gusberti, T.D.H. et al. Monitoramento da multidisciplinaridade no processo de transferência de tecnologia em uma universidade: proposta de análise de cluster. Revista de Administração e Inovação, v.11, n.1, p.309-322, 2014.

Křivánek, M. Computing the nearest neighbor interchange metric for unlabeled binary trees is NP-complete. Journal of Classification, v.3, n.1, p.55-60, 1986.

Linden, R. Técnicas de agrupamento. Revista de Sistemas de Informação da FSMA, v.1, n.4, p.18-36, 2009.

Machado, J.L.M. et al. Formação e seleção de docentes para currículos inovadores na graduação em saúde. Revista Brasileira de Educação Médica, v.35, n.1, p.326-333, 2011.

Metz, J. Interpretação de clusters gerados por algoritmos de clustering hierárquico. 2006. 126f. Dissertação (Mestrado em Ciência da Computação e Matemática Computacional) - Instituto de
Meyer, A.D.S. Comparação de coeficientes de similaridade usados em análises de agrupamento com dados de marcadores moleculares dominantes. 2002. 106f. Dissertação (Mestrado em Agronomia) - Escola Superior de Agricultura Luiz de Queiroz, Universidade de São Paulo, São Paulo, 2002.

Moita, R.M.S. et al. Permanent demand excess as business strategy: An analysis of the Brazilian higher-education market Revista de Administração, v.50, n.1, p.9-25, 2015.

Nassif, V.M.J. et al. Fatores que influenciam na percepção das competências para o exercício da docência. Revista Brasileira de Educação, v.15, n.44, p.364-412, 2010.

Niwattanakul, S. et al. Using of Jaccard Coefficient for keywords similarity. In: Proceedings of the International MultiConference of Engineers and Computer Scientists, 21., 2013, Hong Kong. Hong Kong: IMECS, 2013.

Pachane, G.G. A importância da formação pedagógica para o professor universitário: a experiência da Unicamp. 2003. $268 f$. Tese (Doutorado em Educação) - Faculdade de Educação, Universidade de Campinas, Campinas, 2003.

Piran, F.A.S. et al. Product modularity and its effects on the production process: An analysis in a bus manufacturer. International Journal of Advanced Manufacturing Technology, v.88, n.1, p.2331-2343, 2017.

Reina, D.G. et al. Improving discovery phase of reactive ad hoc routing protocols using Jaccard distance. Journal of Supercomputing, v.67, n.1, p.131-152, 2014.

Seifoddini, $\mathrm{H}$. Single linkage versus average linkage clustering in machine cells formation applications. Computers and Industrial Engineering, v.16, n.3, p.419-426, 1989.

Sharma, S.; Yadav, R.L. Comparative study of K-means and robust clustering. International Journal Advanced Computer Research, v.3, n.3, p.207-210, 2013.

Schonlau, M. The Clustergram: A graph for visualizing hierarchical and non-hierarchical cluster analyses. The Stata Journal, v.3, n.1, p.316-327, 2002.

Voss, C.; Tsikriktsis, N.; Frohlich, M. Case research in operations management. International Journal of Operations and Production Management, v.22, n.2, p.195-219, 2002.

Yin, Y. Application similarity coefficient method to cellular manufacturing. In: Kordic, V. et al. Manufacturing the future. Austria: In Tech, 2006. p.195-258. 